\title{
IKK1 Control of Epidermal Differentiation Is Modulated by Notch Signaling
}

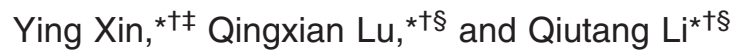 \\ From the James Graham Brown Cancer Center* and the \\ Departments of Ophthalmology and Visual Sciences ${ }^{\dagger}$ and \\ Biochemistry and Molecular Biology, ${ }^{\S}$ University of Louisville \\ School of Medicine, Louisville, Kentucky; and the Key Laboratory \\ of Pathobiology, ${ }^{\ddagger}$ Ministry of Education, Jilin University, \\ Changchun, China
}

The molecular mechanism by which I $\mathrm{BB}$ kinase 1 (IKK1) regulates epidermal differentiation and tumor suppression in the skin is not well understood. As two major regulatory signaling pathways that regulate epidermal homeostasis and differentiation, the p63 and Notch pathways were examined in $I k k 1$ mutant epidermis and keratinocytes. $I k k 1$ inactivation in keratinocytes resulted in increased p63 expression and repression of Notch signaling. The impaired differentiation of $I k k 1^{-/-}$keratinocytes was partially rescued by overexpression of the active form of the Notch1 receptor, the Notch intracellular domain (NICD). In contrast, knockdown of p63 expression by RNA interference was unable to rescue the defect. These results suggest that, in the mammalian skin, IKK1 functions as a differentiation regulator and tumor suppressor through the Notch signaling pathway. (Am J Patbol 2011, 178:1568-1577; DOI: 10.1016/j.ajpath.2010.12.021)

The skin epidermis is a highly organized, stratified structure consisting of a proliferative basal layer and three suprabasal layers (the spinous, granular, and cornified layers). ${ }^{1}$ The basal cells express the skin epithelial progenitor cell markers keratin 5 and 14 ( $\mathrm{K} 5$ and $\mathrm{K} 14$ ), expression of which is turned off once the cells differentiate into the more outer spinous layer cells, which express the K1 and K10 markers. Next to the spinous layer is the granular layer, in which the cells express loricrin, filaggrin, and transglutaminase, which are involved in the assembly of the cornified envelope as the surface barrier of the skin. During the early epidermal development, there is an unique cell type that is intermediate between the transient amplifying (TA) progenitor cells and spinous cells. ${ }^{2}$ These intermediate cells proliferate and express both the progenitor cell marker K14 and the spinous cell marker K1.
$I_{\kappa} \mathrm{B}$ kinase $1(\mathrm{IKK} 1)$ is conventionally considered to be a major component of the IKK complex in the nuclear factor- $\kappa \mathrm{B}(\mathrm{NF}-\kappa \mathrm{B})$ signaling pathway. There is substantial evidence that IKK1 plays a critical role in epidermal development. $^{3-5}$ However, IKK1 regulates keratinocyte proliferation and differentiation by a mechanism that is independent of its kinase activity and NF- $\kappa \mathrm{B}$ activity. ${ }^{6}$ For its function in epidermal development and differentiation, IKK1 must translocate into the nucleus. ${ }^{7}$ A recent study showed that IKK1 is a cofactor for Smad2/3 and is involved in transforming growth factor $\beta$ (TGF $\beta$ )-induced keratinocyte arrest. $^{8}$ Furthermore, IKK1 is proposed to be a tumor suppressor in human and mouse skin, coordinating with the epidermal growth factor receptor (EGFR)/Ras/extracellular signal-regulated kinase (Erk)/EGFR ligand pathway. ${ }^{9}$

Ikk 1 is a downstream target of murine transformation related protein 63 (Trp63, also known as p63), an early epidermal specification gene. ${ }^{10}$ The p63 transcriptional factor and Notch signaling are two major regulatory factors during the early stage of epidermal differentiation. ${ }^{2}$ The p63 protein is necessary for epidermal cell specification and survival, and skin lacking p63 produces only residual $\mathrm{K} 14^{+}$cells. ${ }^{11,12}$ Functioning as a transcription factor, p63 induces and maintains the expression of $\mathrm{K} 14$ and $\mathrm{K} 5$ in the proliferating epidermal progenitor cells. ${ }^{13,14}$ Subsequently, p63, in collaboration with the Notch signal pathway, induces expression of the later stage markers K1 and K10. Reciprocal regulation of p63 and Notch1 has been postulated in balancing keratinocyte proliferation and differentiation. ${ }^{15,16}$

The Notch signaling pathway is a highly conserved signal transduction system. ${ }^{17}$ There are four Notch receptors in vertebrates (Notch1 to Notch4) and five Notch ligands [Jagged1, Jagged2, and Delta-like 1 (D\|1), DII3, and DII4]. Once activated by ligands, Notch is cleaved by $\gamma$-secretase to release the active Notch intracellular domain (NICD), leading to translocation of the NICD into the nucleus. NICD

Supported in part by grants NIH/NCRR COBRE 5 P20 RR018733 (Q. Li), 5 P20 RR017702 (Q. Lu), NIH R01-EY019891 (Q. Li), and EY018830 (Q. $\mathrm{Lu}$ ); by Research to Prevent Blindness (for general support); and $\mathrm{NIH}$ EY015636 (funding of Histocore).

Accepted for publication December 17, 2010.

Address reprint requests to Qiutang Li, Ph.D., Department of Ophthalmology and Visual Sciences, University of Louisville, $301 \mathrm{E}$ Muhammad Ali Blvd, Louisville, KY 40202. E-mail: q.li@louisville.edu. 
associates with the transcription factor recombination signal-binding protein- $\mathrm{J} \kappa(\mathrm{RBP}-\mathrm{J} \kappa)$ to generate a transactivation complex that activates transcription of the target genes, such as the hairy/enhancer of split (Hes) transcriptional repressors. ${ }^{17}$ In the mouse epidermis, Notch1 and Notch2 are strongly expressed in the spinous layer and Notch ligands are expressed in patterns overlapping with Notch. ${ }^{18-20}$

Conditional ablation of Notch signaling by deletion of genes encoding RBP- $J \kappa,{ }^{21}$ both Notch1 and Notch2, ${ }^{22}$ or Hes $1^{23}$ suggests that Notch induces commitment to a spinous cell fate through a few separate, but functionally relevant, events, such as i) RBP-J $\kappa^{-}$- and Hes1dependent induction of $\mathrm{K} 1$ and $\mathrm{K} 10$, ii) cell cycle arrest caused by the RBP- $\mathrm{J}_{\kappa}$ - and Hes1-dependent induction of $\mathrm{p} 21^{19}$, iii) basal gene repression through RBP-Jk-dependent and Hes1-independent suppression of $\mathrm{K} 14$ and $\mathrm{K} 5$, and iv) promotion of further differentiation into granular cells via RBP- $J \kappa^{-}$and Hes1-independent induction of involucrin. ${ }^{19}$ The function of Notch in epidermal homeostasis was further confirmed by studies in mice that have constitutively activated Notch1 in the epidermis. ${ }^{22-25} \mathrm{Hy}-$ perproliferation of the basal keratinocytes caused by postnatal ablation of Notch1 suggests that Notch1 functions as a tumor suppressor gene in the mammalian skin. ${ }^{26}$ Notch suppression of keratinocyte proliferation is considered to be cell-autonomous ${ }^{27}$; however, a recent study suggested that Notch1 functions as a tumor suppressor via a nonautonomous mechanism. ${ }^{28}$

In the present study, we examined expression of p63 and Notch and their signaling activities in $I k k 1^{-1-}$ keratinocytes and investigated their contribution to the $1 \mathrm{kk} 1^{-1-}$ phenotype. Our data showed that significantly reduced Notch activity contributes to the undifferentiated phenotypes of $I k k 1^{-1-}$ keratinocytes.

\section{Materials and Methods}

\section{Animals, Animal Care, and Genotyping}

All animal protocols used in these studies were approved by the University of Louisville Institutional Animal Care and Use Committee (IACUC). The generation of the Ikk1 knockout mice was performed as reported previously. ${ }^{4}$ $\mid k k 1^{+/-}$heterozygous mice on the C57BL/6J background were bred to generate homozygous mutants. To quantitate cell proliferation in embryos, the pregnant mice were injected with 5-bromo-2'-deoxyuridine (BrdU) (150 $\mathrm{mg} / \mathrm{kg}$ body weight) for 90 minutes before collection of the embryos at embryonic days E11.5, E12.5, E13.5, E14.5, E16.5, E17.5, and E18.5. The embryos were fixed overnight in $4 \%$ cold paraformaldehyde. The tissues derived from the yolk sac of the embryos were saved and processed for PCR genotyping. ${ }^{4}$

\section{Histology and Immunolabeling}

Tissues were paraffin-embedded and cut into sections $7-\mu \mathrm{m}$ thick, deparaffinized, and stained with hematoxylin and eosin. For most of the immunostaining reactions, the tissues or cell slides were heated at $90^{\circ} \mathrm{C}$ for 20 minutes in $10 \mathrm{mmol} / \mathrm{L}$ Tris-EDTA buffer (pH 9.0). For immunostaining, the following primary antibodies were used: mouse anti-p63 (1:200, catalog no. sc-8431), goat anti- $\Delta$ Np63 (1:200, sc-8609), and rabbit anti-p63 $\alpha$ (1:200, sc-8344) from Santa Cruz Biotechnology (Santa Cruz, CA); rat anti-bromodeoxyuridine (BrdU; 1:800, MAS 250c) from Harlan-Sera Lab (Belton Loughborough, Leicestershire, England); mouse anti-K10 (1:500, PRB-159S), rabbit antiK14 (1:500, PRB-155P), rabbit anti-loricrin (1:500, PRB145P), and rabbit anti-involucrin (1:500, PRB-140P) from Covance Research Products (Denver, PA); and rabbit anti-zonula occludens-1 (ZO-1; 1:600, catalog no. 617300) from Zymed Laboratories (San Francisco, CA). The secondary carbocyanine 3 (Сy3)-conjugated and fluorescein isothiocyanate (FITC)-conjugated antibodies were obtained from Jackson ImmunoResearch Laboratories (West Grove, PA).

\section{Keratinocyte Culture and Reagents}

To prepare keratinocytes, the full-thickness skin taken from E18.5 embryos was digested overnight at $4{ }^{\circ} \mathrm{C}$ with $0.25 \%$ trypsin. The wild-type (WT) epidermal layer was peeled from the dermis, and keratinocytes were isolated from the inner surface by scraping cells from the epidermal sheet. The $l k k 1^{-1-}$ keratinocytes were scraped directly from the outside surface of the skin after overnight trypsin digestion. Keratinocytes were cultured in keratinocyte serum-free media (SFM; Invitrogen, Carlsbad, $\mathrm{CA})$. For BrdU labeling assays, the cells were incubated in the medium containing $10 \mu \mathrm{mol} / \mathrm{L} \mathrm{BrdU}$ for 90 minutes and then were fixed with $4 \%$ paraformaldehyde for 15 minutes before the immunostaining with anti-BrdU antibody.

\section{Calculation of $\mathrm{BrdU}^{+}$and $\mathrm{p} 63^{+}$Cells and Statistical Analysis}

The numbers of $\mathrm{BrdU}^{+}$cells and the total DAPI-positive cells on each section were counted on a digital photo taken at $30 \times$ magnification. We counted the average number of $\mathrm{BrdU}^{+}$cells in a defined length $(600 \mu \mathrm{m})$ of epidermis from $\geq 6$ sections of each group. The percentile of $\mathrm{BrdU}^{+}$or $\mathrm{p} 63^{+}$cells in lentiviral transduced cells as labeled by green fluorescent protein (GFP; 1000 cells) were counted and calculated from three independent experiments. All data are shown as means \pm SD. The differences were considered statistically significant if the $P$ values were $<0.05$.

\section{Lentiviral Vector and Viral Production}

The p63 shRNA sequence 5'-GAGGTTGTGAAACGATGC-3' used was taken from an article by Nguyen et al. ${ }^{16}$ A control (scrambled shRNA cloned into L-CMVGFP-Nhel) was also used. We cloned the shRNAs into the cytomegalovirus-green fluorescent protein lentiviral vector L-CMV-GFP-Nhel (kindly provided by Dr. Inder Verma) using a PCR approach, expression being driven by the human $\mathrm{H} 1$ promoter. The detailed plasmid con- 
struction procedure used was as described previously. ${ }^{29,30}$ The lentiviral vectors EF.hiCN1.CMV.GFP and EF.mHES1.CMV.GFP were obtained from Addgene (Cambridge, MA). Lentiviruses were produced by a three-plasmid (for second-generation lentiviral vectors) or four-plasmid (for third-generation lentiviral vectors) transfection system, as described by Tiscornia et al. ${ }^{30}$

\section{Transfection and Dual-Luciferase Assays}

Dual-luciferase assays were performed using an assay system from Promega (Madison, WI). Notch transcription activity was measured using a Cignal RBP-Jk reporter luciferase kit (catalog no. CCS-014L; SA Biosciences, Frederick, MD). The firefly luciferase reporter gene under the control of a minimal CMV promoter and tandem repeats of the RBP-Jk transcriptional response element was used as an experimental reporter, and renilla luciferase plasmid-thymidine kinase ( $\mathrm{pRL}-\mathrm{TK}$ ) was cotransfected as an internal control. Luciferase activity was expressed as the ratio of firefly luciferase activity to renilla luciferase activity.

\section{RNA Isolation and Quantitative PCR}

Primary keratinocyte cultures at $70 \%$ to $90 \%$ confluence were used for RNA extraction. Total RNA was extracted using TRIzol reagent (Invitrogen). The A260/A280 ratio of all RNA samples was $>2.0$, and the RNA quality was checked on a $1 \%$ agarose gel. Double-stranded cDNA was reverse-transcribed using random primers and a SuperScript VILO cDNA synthesis kit (Invitrogen). Quantitative PCR (qPCR) was performed according to the manufacturer's instructions (Stratagene M×3000P qPCR system; Agilent Technologies, Santa Clara, CA) using primer sets for actin as an internal standard. The primers for mouse Notch1 (ID 13177625a1), Notch2 (ID 33859592a3), Notch3 (ID 6679096a1), Notch4 (ID 6754874a1), DII1(ID 6681197a3), DII4 (9506547a1), Jagged1 (ID 7305197a1), Jagged2 (ID 2765404a1), K1 (12859782a1), Involucrin (ID 6680506a1), and Filaggrin (ID 20750113a1) were designed based on the online PrimerBank database (Harvard Medical School, Boston, MA; http://pga.mgh.harvard.edu/primerbank). The qPCR primer sequences used for Hes 1, Hey 1, and p21 were as reported previously. ${ }^{31}$

\section{Western Blot Analysis}

Keratinocytes were lysed in cold radioimmunoprecipitation assay buffer $(20 \mathrm{mmol} / \mathrm{L}$ Tris- $\mathrm{HCl}, \mathrm{pH} 7.4,100$ $\mathrm{mmol} / \mathrm{L} \mathrm{NaCl}$, and $0.2 \%$ each of deoxycholate, Triton X-100, and Nonidet P-40) containing a protease-inhibitor mixture (Roche Diagnostics, Indianapolis, IN). Equal amounts of whole-cell lysates were separated on 10\% SDS polyacrylamide gel and were transferred onto a nitrocellulose membrane. For immunoblotting, we used the following primary antibodies: mouse anti-p63 (1:200, catalog no. Sc-8431), goat anti-C terminus of $14-3-3 \sigma$ (1:200, catalog no. sc-7683), goat anti-14-3-3 $\sigma$ (1:200, catalog no. sc-7681), rabbit anti-Notch1 full-length (1:200, catalog no. sc-9170), and goat anti-Notch 1 (1:200, catalog no. sc-23304) from Santa Cruz Biotechnology; rabbit polyclonal anti-cleaved Notch-1 (1:200, ab8925) from Abcam (Cambridge, MA); and mouse anti- $\beta$-actin (1: 1000, A2228) from Sigma-Aldrich (St. Louis, MO). The secondary antibodies were conjugated with horseradish peroxidase (Amersham Pharmacia, Piscataway, NJ). Blots were visualized using an enhanced chemiluminescence system (ECL; Amersham Pharmacia).

\section{Results}

\section{IKK1 Is Required for the Commitment of Proliferative Intermediate Cells to the Spinous Cell Type during Development}

Normal epidermal specification and stratification occur between days E8.5 and E18.5. ${ }^{2}$ To investigate how loss of Ikk1 affects epidermal morphogenesis, we performed immunohistochemical staining on developing epidermis from WT and $l k k 1^{-l-}$ embryos (Figure 1). The epidermal basal layer contained a monolayer of proliferating $\mathrm{K} 14^{+}$ cells (Figure 1A). The proliferating intermediate cells were differentiated and became the first suprabasal layer at E13.5. These cells were capable of proliferation and still expressed the progenitor cell marker K14, but also started to express $\mathrm{K} 10$, conventionally a marker of spinous cells (Figure 1, A and B). These intermediate cells existed for only a period of 2-3 days. By E15.5, they had exited the cell cycle, terminated K14 expression, and differentiated into more advanced spinous cells (Figure 1A).

At the same time, the granular layer, recognized by the specific marker loricrin, appeared above the spinous layer (Figure 1C). The first recognized phenotype in the $1 \mathrm{k} k 1^{-1-}$ epidermis appeared at E13.5, with the expansion of the intermediate cell layer containing proliferative $\mathrm{K}_{14}{ }^{+}$and $\mathrm{K}_{10} 0^{+}$cells (Figure $1, \mathrm{~A}$ and $\mathrm{B}$ ). The expansion of the intermediate cell layer was due to increased cell proliferation, as demonstrated by increased BrdU incorporation at E13.5 (Figure 1, D and E). The suprabasal cells remained in the epidermal progenitor stage and did not differentiate further into spinous and granular cell layers (Figure 1C). These findings indicate that IKK1 is essential for the intermediate cell layer to differentiate into the spinous layer during embryonic development.

\section{Increased p63 Expression in Ikk $1^{-1-}$ Keratinocytes Contributes to the Hyperproliferation Phenotype but Not to Defective Differentiation}

We observed the hyperproliferative epidermis at as early as E13.5 in the $1 k k 1^{-1-}$ embryos (Figure 1, D and E). To determine whether p63 participated in the development of the $1 \mathrm{k} \mathrm{K}^{-1-}$ epidermal phenotype, we investigated p63 expression by immunohistochemistry. The p63 protein was expressed by almost all epithelial cells in the $1 k k 1^{-I-}$ epidermis, whereas its expression was restricted 
$A$

Figure 1. Developmental defects in the $I k k 1^{-1-}$ epidermis. A: Immunofluorescence study using an anti-K14 antibody (red) and nuclear staining with DAPI (blue) on skin from embryos at six embryonic days, E12.5-E17.5. B: Immunofluorescence study using anti-K10 antibody and DAPI on skin from E14.5 embryos. C: Immunofluorescence study using anti-loricrin antibody and DAPI on skin at E15.5 and E16.5. D: Immunofluorescence study using anti-BrdU antibody and DAPI on skins at E11.5, E12.3, and E13.5. The pregnant mice were injected with 150 $\mathrm{mg} / \mathrm{kg}$ body weight of BrdU and embryos were collected 90 minutes later. Scale bar $=50 \mu \mathrm{m}$ (A-D, all images). E: Quantification of BrdU ${ }^{+}$ cells in the developing epidermis. The number of the $\mathrm{BrdU}^{+}$cells per $600 \mu \mathrm{m}$ of fixed length parallel to the skin surface. Data are reported as means $\pm \mathrm{SD}$ of three independent experiments. ${ }^{* *} P<0.01$, two-tail $t$-test.

to the basal epidermal progenitor cells in the WT control (Figure 2A).

Tumor protein p63 is expressed by two different promoters, which generate two classes: TAp63, which includes the N-terminal transactivation domain, and $\Delta \mathrm{Np63}$, which lacks this domain. Each class comprises three different isoforms, generated in distinct splicing processes. ${ }^{32}$ Whereas the TAp63 isoforms initiate epithelial stratification, the $\Delta \mathrm{Np} 63$ isoforms appear to control the terminal differentiation of the epidermis. ${ }^{32}$ Of these six isoforms, $\Delta \mathrm{Np} 63 \alpha$ was the major isoform overexpressed in $\mid k k 1^{-1-}$ skin, as demonstrated by specific antibody immunostaining (Figure 2B) and by Western blotting, which showed the same molecular weight as that of the $\Delta$ Np63 $\alpha$ control (Figure 2C).

$\Delta \mathrm{Np} 63 \alpha$ maintains both $\mathrm{K} 14$ expression and intermediate cell identity, and p63 overexpression attenuates the expression of the late differentiation markers loricrin and filaggrin. ${ }^{14}$ To determine whether the increased $\Delta N p 63 \alpha$
B
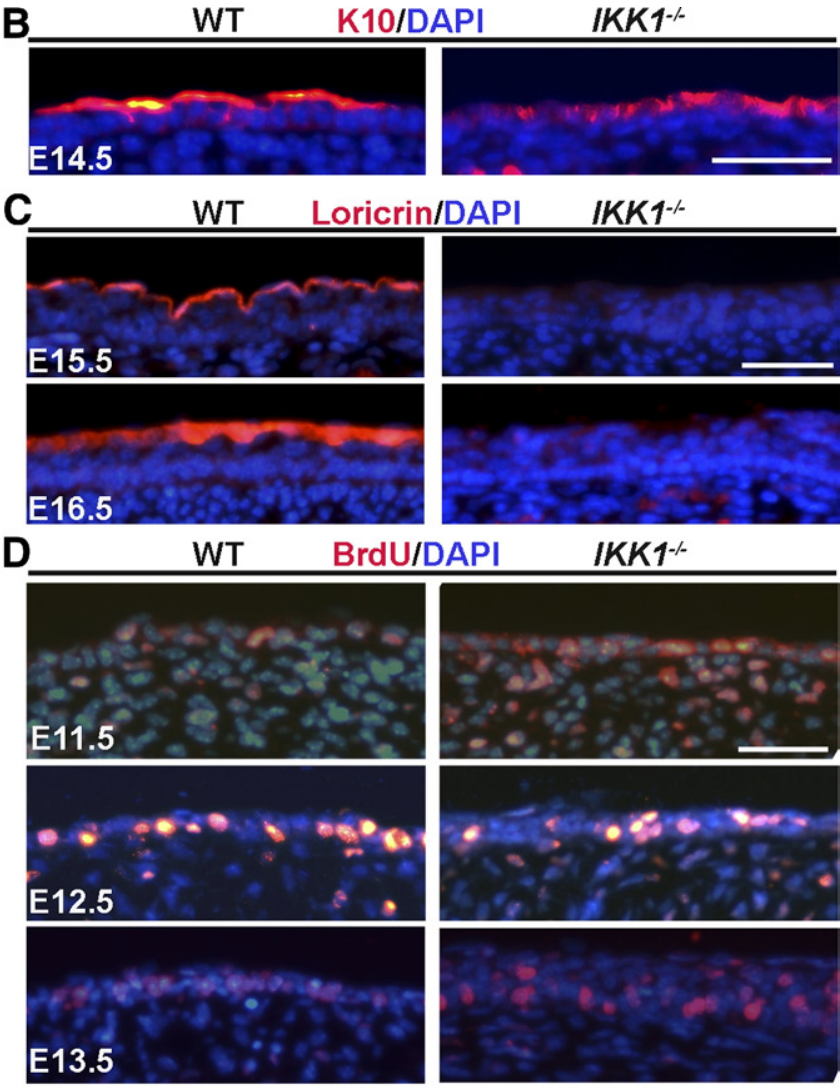

E

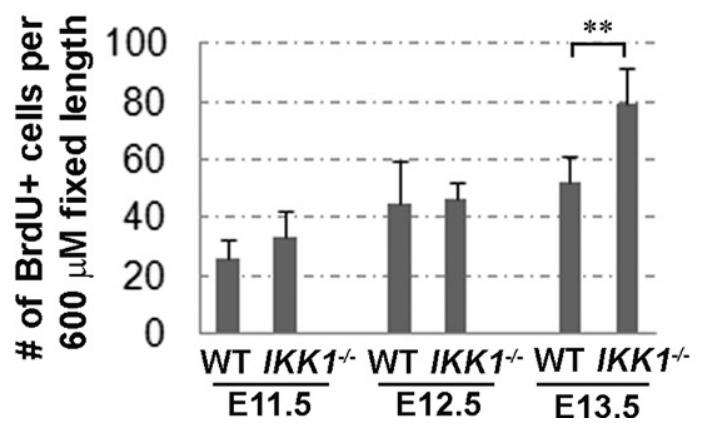

expression was responsible for unsustained proliferation and defective differentiation, we examined $I k k 1^{-1-}$ keratinocyte differentiation after knockdown of p63 expression using the short hairpin shRNA method with a lentiviral shRNA vector containing the p63-specific interference sequence. Expression of p63 was efficiently blocked (Figure 2D). The BrdU-labeling assay showed that p63 knockdown reduced mitotic activity equally in WT and $1 k \mathrm{k} 1^{-1-}$ keratinocytes, compared with the relevant controls transduced with lentiviruses expressing scrambled shRNA (Figure 2E). Transduced cells could be easily detected, because the lentiviral vectors coexpressed a GFP reporter.

Next, we examined the effect of p63 down-regulation on $\mid k k 1^{-1-}$ keratinocyte differentiation. When cultured in medium containing a low level of calcium (0.02 mmol/L), keratinocytes remain undifferentiated, but, shortly after switching to high calcium $(0.5 \mathrm{mmol} / \mathrm{L})$, they exit from the cell cycle and start to differentiate. The early event fol- 
A

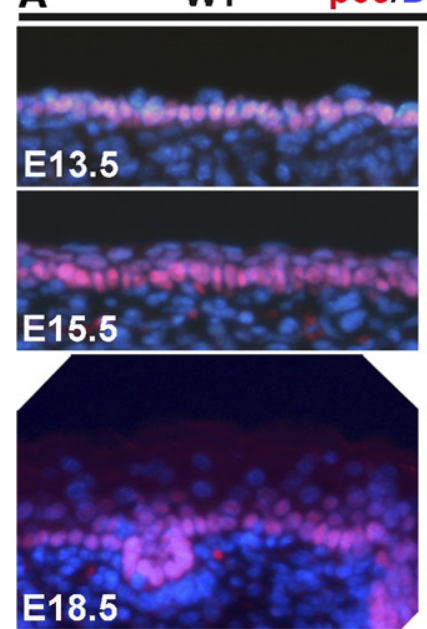

E
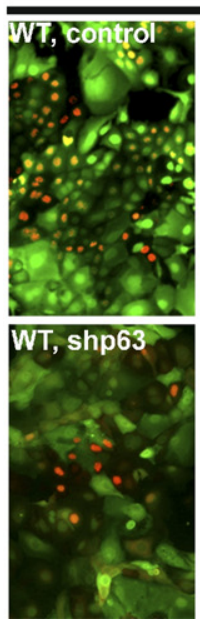

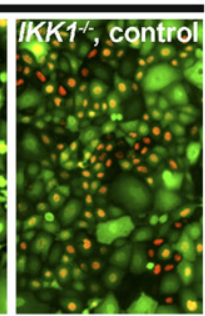

IKK1 1 , shp63

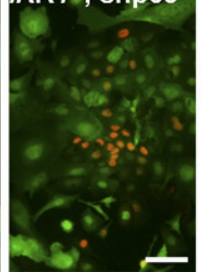

IKK1 ${ }^{-1}$
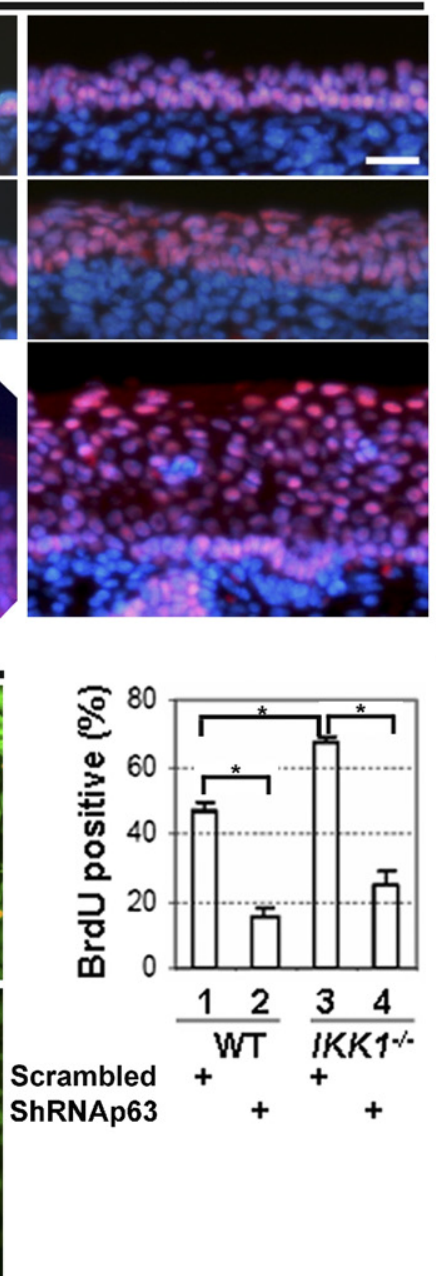

B

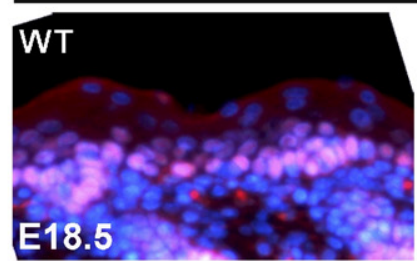

C

\section{p63}

actin

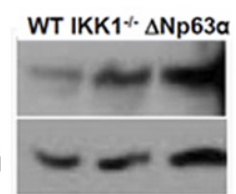

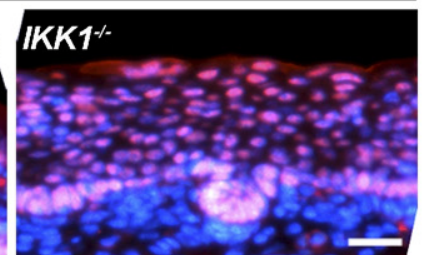

D

p63

actin

Scrambled ShRNAp63

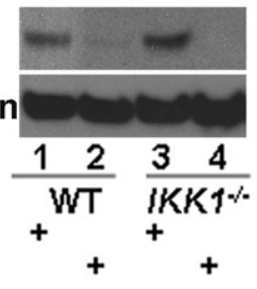

G

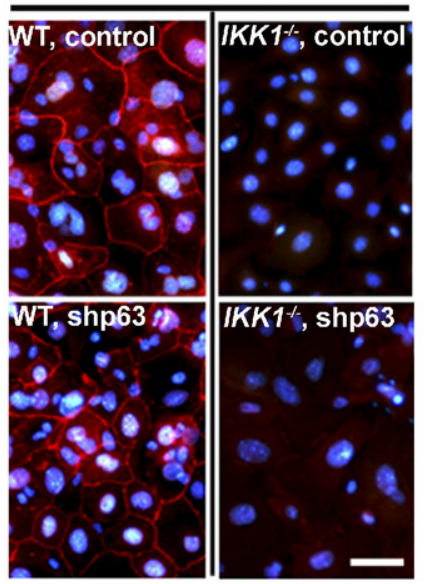

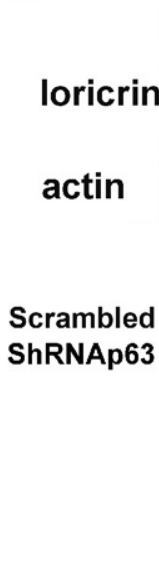
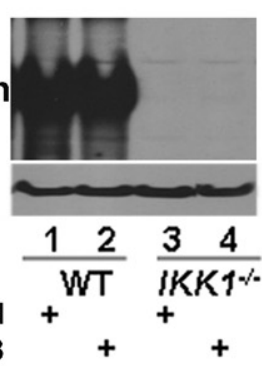

Figure 2. Expression of $\mathrm{p} 63 \alpha$ is increased in the $I k k 1^{-1-}$ epithelium, and knockdown of p63 is not able to rescue $I k k 1^{-/-}$differentiation. A: Immunohistochemistry of skin sections from embryos at days E13.5, E15.5, and E18.5, stained with anti-p63 antibody (red) and DAPI. B: Immunohistochemistry of skin sections from day E18.5 embryos stained with anti-p63 $\alpha$ antibody (red) and DAPI. C: Western blot analysis of protein lysates from primary WT, Ikk $1^{-/-}$keratinocytes, and the $293 \mathrm{~T}$ cells transfected with mouse $\Delta$ p63 $\alpha$ cDNA $(68 \mathrm{kDa})$ for p63 expression using anti-p63 antibody. D: shRNA knockdown of p63 expression in the primary WT and $I k k 1^{-1-}$ keratinocytes transduced with lentiviruses expressing p63 shRNA. E: Decreased cell proliferation in both WT and $I k k 1^{-1-}$ keratinocytes transduced by the p63-specific shRNA lentivirus. Primary WT and $I k k 1^{-1}$ - keratinocytes were transduced with either p63-specific or scrambled shRNA lentiviruses for 3 days; cell proliferation was then measured by BrdU incorporation. The micrographs show the merged images for BrdU immunostaining (red) and GFP (green). The bar chart shows the percentage of $\mathrm{BrdU}^{+}$cells in the total transduced cell population (GFP ${ }^{+}$). Error bars indicate the SD for three independent experiments. ${ }^{*} P<0.001$. F: ZO- 1 immunohistochemistry was performed on $0.5 \mathrm{mmol} / \mathrm{L}$ calcium-induced differentiated WT or $I k k 1^{-/-}$keratinocytes that had been previously treated with or without p63 shRNA lentiviruses. G: Western blot analysis was performed on the cell lysates of the p63 knockdown WT and $I k k 1^{-1-}$ keratinocytes cultured in $0.5 \mathrm{mmol} / \mathrm{L}$ calcium of differentiation-inducing medium, to examine loricrin ( $\left.38 \mathrm{kDa}\right)$ expression. Scale bars: $50 \mu \mathrm{m}(\mathbf{A}$, $\mathbf{B}$, and $\mathbf{F}) ; 150 \mu \mathrm{m}(\mathbf{E})$.

lowing the calcium-induced keratinocyte cell differentiation is the tight junction formation, associated with increased and more continuous staining of ZO-1 at the cell-cell borders. ${ }^{33,34}$ Wild-type differentiating keratinocytes developed bell-like tight junctions between neighboring cells, as revealed by the strong immunoreactivity for the tight junction-associated ZO-1 protein (Figure 2F). After high-calcium induction, however, $I k k 1^{-1-}$ keratinocytes never formed a continuous $\mathrm{ZO}-1^{+}$bell-like tight junction, only occasionally developing some sporadic spots stained weakly with anti-ZO-1 antibody (Figure 2F). Thus, inhibition of p63 overexpression by shRNA in $\| k k 1^{-1-}$ cells was unable to rescue the $\mid k k 1^{-1-}$ differentiation defect. This conclusion was further validated by the lack of calcium-induced loricrin (Figure 2G) and filaggrin (data not shown) expression in p63 knockdown $1 k k 1^{-1-}$ keratinocytes.

\section{IKK1 Deficiency Reduces Notch Transcription and Notch Activity}

Inactivation of Notch1 in young mice induces hyperproliferation of the basal epidermal layer and deregulates expression of multiple differentiation markers. ${ }^{26} \mathrm{We}$ therefore examined Notch1 activity in $\mid k k 1^{-1-}$ keratinocytes. Western blot analysis showed a reduction in NICD levels in $l k k 1^{-1-}$ keratinocytes (Figure $3 \mathrm{~A}$ ). It is well documented that the NICD converts the ubiquitous transcription factor RBP-J $\mathrm{k}$ from a repressor to an activator 


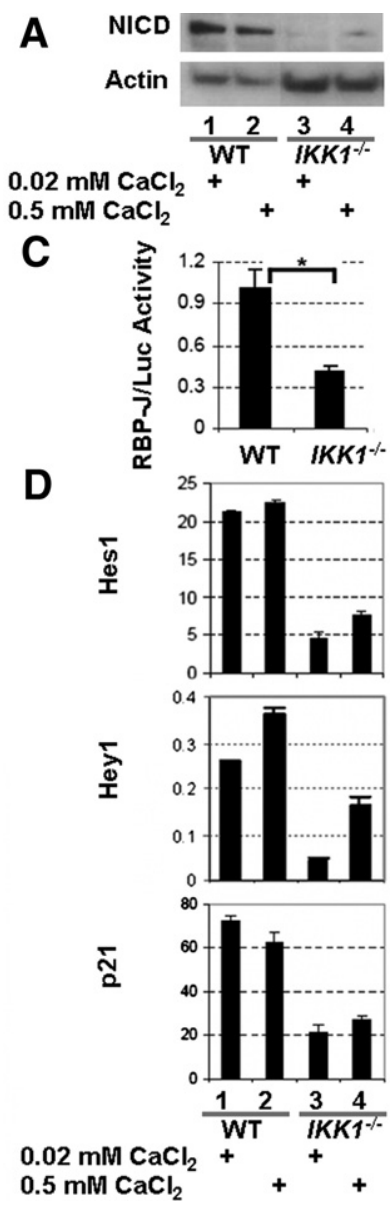

B
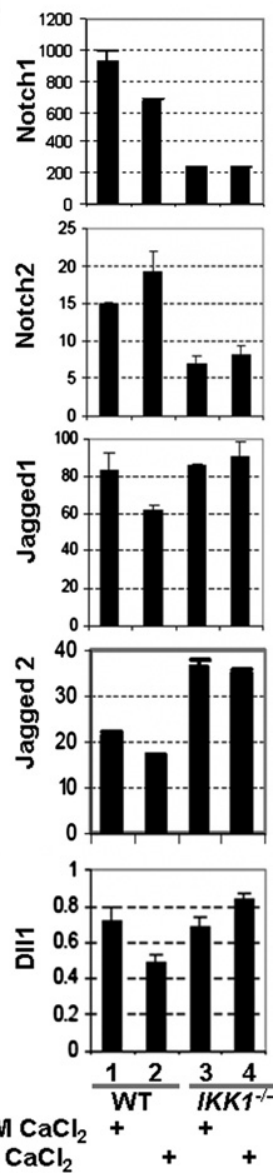

Figure 3. Reduced Notch activity in $I k k 1^{-1-}$ keratinocytes. A: Impaired NICD production in $I k k 1^{-1-}$ keratinocytes, as demonstrated by Western blotting. Primary WT and $I k k 1^{-1-}$ keratinocytes were cultured in medium containing $0.02 \mathrm{mmol} / \mathrm{L}$ or $0.5 \mathrm{mmol} / \mathrm{L}$ calcium for 48 hours. Western blots were probed by NICD $(110 \mathrm{kDa})$ and actin $(42 \mathrm{kDa})$ antibodies. B: qPCR measurement of Notch receptors and ligands in WT and $I k k 1^{-1-}$ keratinocytes incubated in low- and high-calcium medium for 48 hours. C: Decreased RBP-J $\kappa$ transcription activity in $I k k 1^{-1-}$ keratinocytes as demonstrated by RBP-J $\kappa$-luciferase assay. The primary keratinocytes were cotransfected with a Notch-responsive reporter (RBP-J $\kappa$-firefly luciferase) and the $\mathrm{CMV} /$ renilla luciferase vectors for 48 hours; the cell lysates were subsequently subjected to firefly and renilla luciferase activity measurement. Promoter activity is expressed as arbitrary units using the renilla reporter for internal normalization. Each condition was tested in triplicate wells. Error bars indicate SD. * $P$ $<0.001$. D: qPCR measurement of the mean relative mRNA levels for the Notch targets Hes1, Hey1, and p21. The WT and $I k k 1^{-1-}$ keratinocytes were incubated in low- and high-calcium medium for 48 hours before they were subjected to mRNA preparation and qPCR quantification. Error bars indicate SD

through a mechanism of dissociation of nuclear corepressors and recruitment of coactivators. ${ }^{17}$ We therefore examined Notch signaling activity by performing a Notch reporter luciferase assay. As expected, RBP-Jк reporter activity was significantly lower in the $l k k 1^{-1-}$ cells than in the WT control (Figure 3C).

Notch signaling has been shown to trigger a cascade of transcription of downstream target genes, including Hes 1, Hey1, and p21. To further evaluate Notch activity, we examined the level of expression of Hes 1 , Hey1, and $p 21$ in $\mid k k 1^{-1-}$ keratinocytes by-real time qPCR. Consistent with the diminished Notch signaling, the transcription of these Notch target genes was sig-

nificantly down-regulated in $\mid k k 1^{-1-}$ keratinocytes (Figure 3D).

To understand the causes of the impaired Notch activity, we examined the expression of all known Notch receptors and ligands. Here, we show the qPCR analysis data only for Notch1, Notch2, Jagged1, Jagged2, and DIl1, because we failed to detect expression of the other receptors and ligands. We found that reduced NICD activity correlated with reduced transcription of the receptors Notch1 and Notch2, but not of the ligands Jagged1, Jagged2, and Dll1 (Figure 3B).

\section{Overexpression of Notch1 Induces Differentiation of $\mathrm{lkk} 1^{-1-}$ Keratinocytes}

To test whether Notch signaling functioned downstream of the IKK1 molecule in the regulation of keratinocyte differentiation, we transduced both WT and mutant keratinocytes with NICD-expressing lentiviruses or control lentiviruses, both coexpressing a GFP reporter vector. After culture in high-calcium medium, WT keratinocytes expressing either GFP or NICD/GFP were induced to differentiate, as demonstrated by expression of the granular cell markers loricrin and filaggrin (data not shown) and by the formation of tight junctions expressing the ZO-1 marker (Figure 4A). In contrast, $1 \mathrm{kk} 1^{-1-}$ keratinocytes transduced with GFP did not respond to high-calcium induction, and the cell membrane between the two adjacent cells expressed only a weak, spotted ZO-1 signal pattern (Figure 4A). Of note, transduction with the NICD-expressing vector, indicated by the simultaneous expression of GFP, not only inhibited keratinocyte proliferation, as demonstrated by decreased BrdU incorporation (Figure 4B, columns 7 and 8 versus columns 5 and 6) and p63 expression (Figure 4C, columns 7 and 8 versus columns 5 and 6 ), but also enhanced spinous cell differentiation, as demonstrated by induction of involucrin (Figure 4D, columns 7 and 8 versus columns 5 and 6 , and Figure 4G). More strikingly, ectopic expression of NICD induced tight junction formation in response to high-calcium induction (Figure 4A), suggesting that Notch signaling is a major downstream target of IKK1 in the promotion of epidermal differentiation. However, the overexpressed NICD was unable to support full terminal differentiation of $\mid k k 1^{-1-}$ keratinocytes into granular cell types, as demonstrated by lack of filaggrin expression (Figure 4E, columns 5-8), whereas high calcium induced filaggrin expression in WT keratinocytes, even in the absence of exogenous NICD (Figure 4E, columns 1-4). Functional overexpression of NICD was validated by the induction of its target gene, Hes 1 (Figure 4F, columns 3 and 4 versus columns 1 and 2 , and columns 7 and 8 versus columns 5 and 6).

\section{Notch1, but Not Hes1, Promotes Ikk1 ${ }^{-1-}$ Keratinocyte Differentiation}

Hes 1 is one of the major targets for Notch signaling, but whether Notch signaling promotes spinous cell commitment and induces terminal differentiation into granular 
A
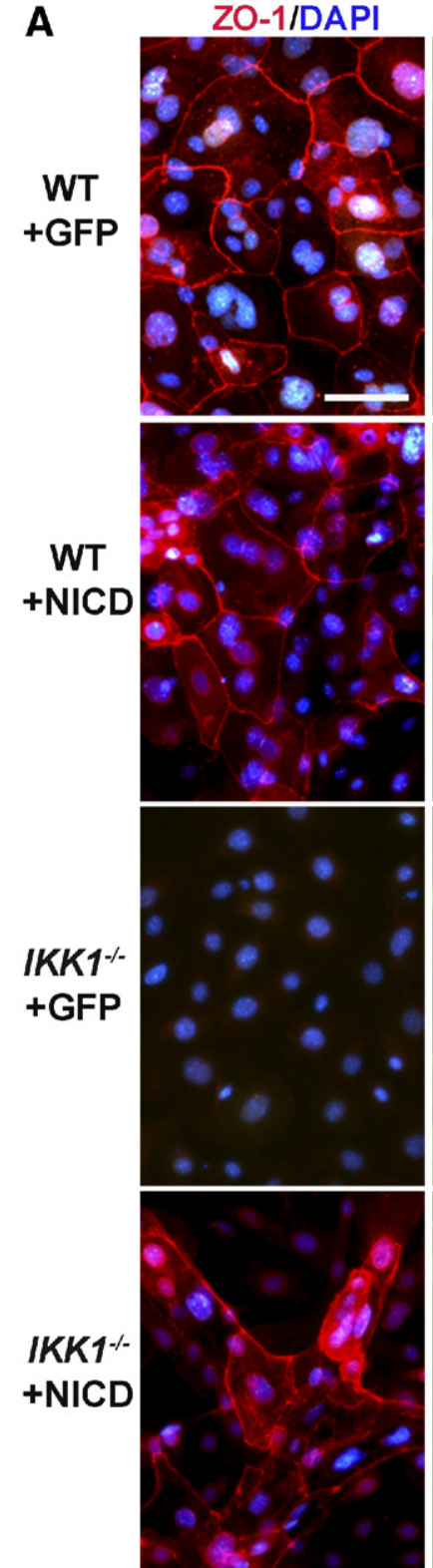

GFP/DAPI
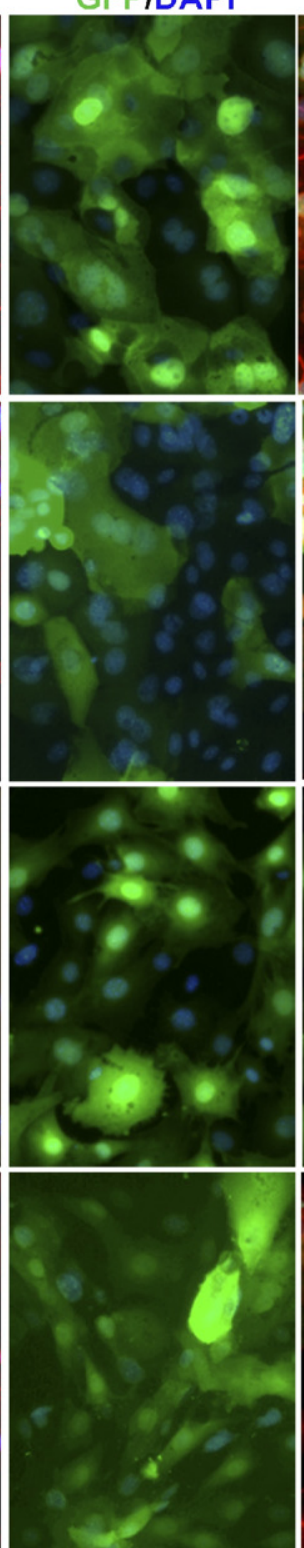

ZO-1/GFP/DAPI
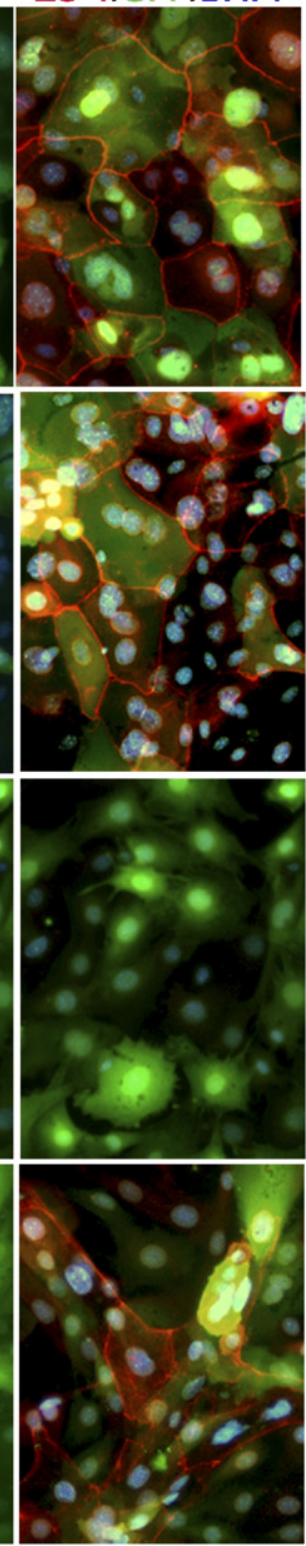

B

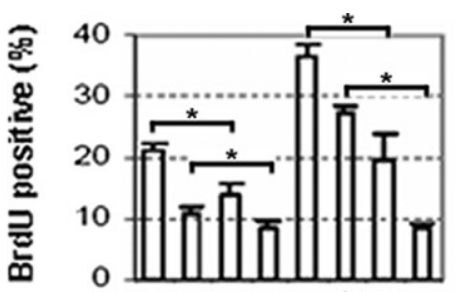

C

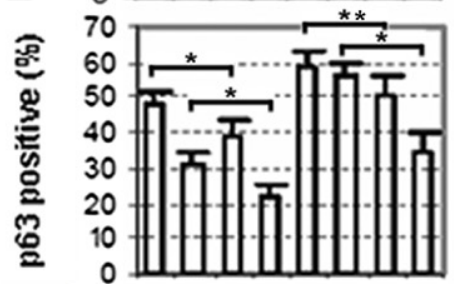

D

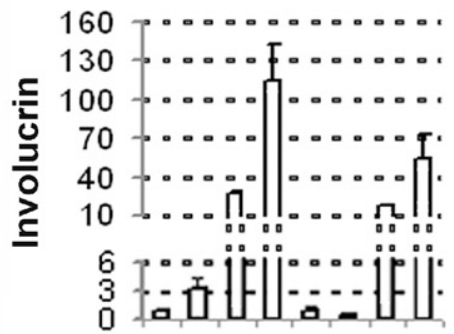

E

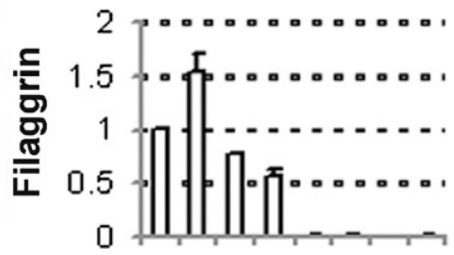

$\mathbf{F}$

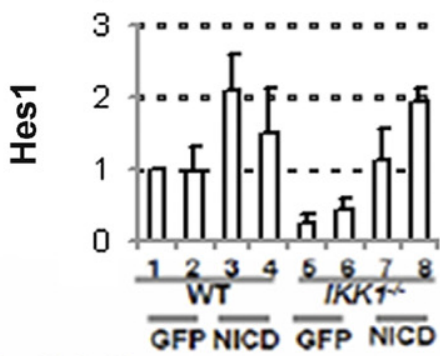

G

Involucrin/DAPI

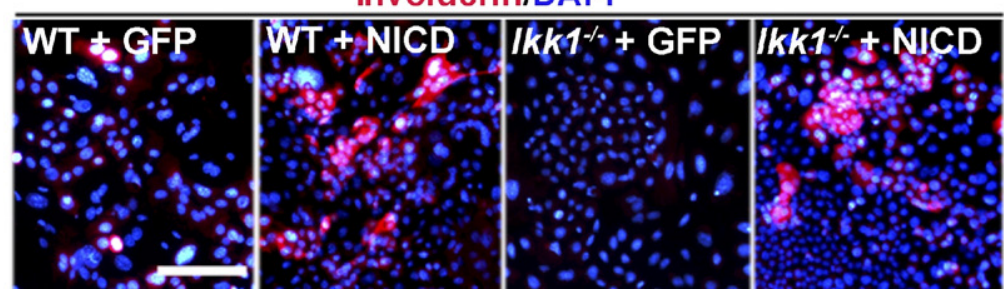

Figure 4. Ectopical overexpression of NICD restores calcium-induced tight junction formation in running into each other. AEC Ikk1 ${ }^{-/-}$keratinocytes. A: Primary WT and $I k k 1^{-1-}$ keratinocytes were transduced with lentiviruses expressing either NICD or GFP for 24 hours and were subsequently cultured in $0.02 \mathrm{mmol} / \mathrm{L}$ or $0.5 \mathrm{mmol} / \mathrm{L} \mathrm{Ca}^{2+}$ medium for an additional 48 hours. Left: The newly formed tight junction induced by $0.5 \mathrm{mmol} / \mathrm{L}$ calcium was visualized by ZO-1 staining (red) and the nuclei were counterstained by DAPI (blue). Center: Transduced cells as shown by lentiviruses expressing GFP or NICD/GFP were visualized by green fluorescence. Right: Merged images. Scale bar $=50 \mu \mathrm{m}$ (all images). B and C: Quantification of BrdU incorporation (B) and p63 expression $(\mathbf{C})$ for WT and $I k k 1^{-1-}$ keratinocytes expressing NICD or GFP control. Error bars represent SD for three independent experiments. ${ }^{*} P<0.001$; ${ }^{* *} P<0.01$. D-F: Transcription levels of the spinous cell marker gene involucrin $(\mathbf{D})$, granular cell marker gene filaggrin $(\mathbf{E})$, and the Notch target gene Hes1 (F) were detected by qPCR. Data are reported as means \pm SD of three independent experiments. G: Involucrin immunostaining on the primary WT and $I k k 1^{-/-}$keratinocytes transduced with lentiviruses expressing either NICD or GFP. Cells were cultured in 0.5 mmol/L Ca ${ }^{2+}$ medium for 48 hours. Scale bar $=150 \mu \mathrm{m}$ (all images). 


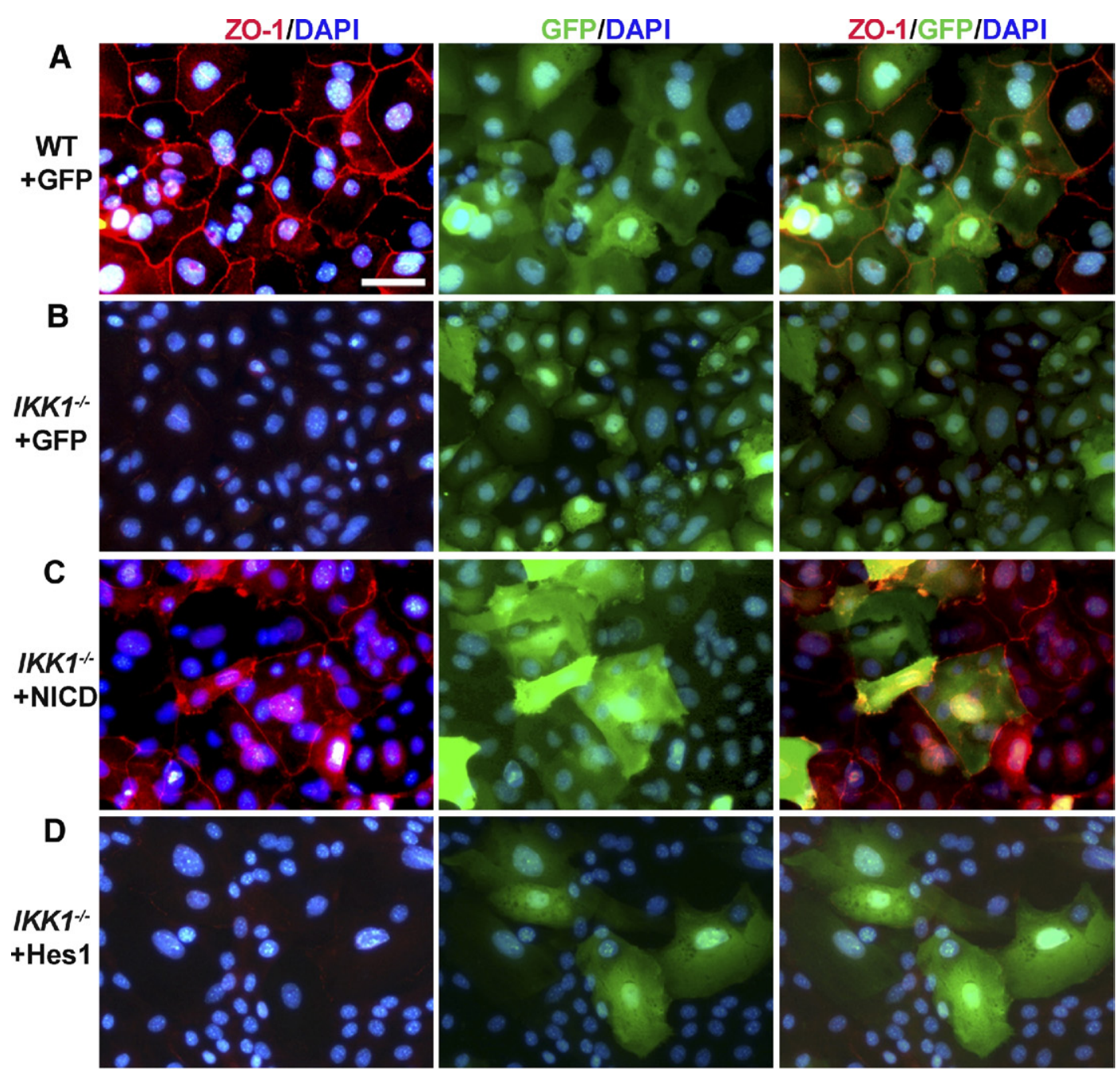

$\mathbf{E}$

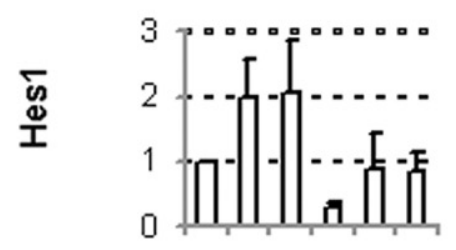

든
$\mathbf{F}$

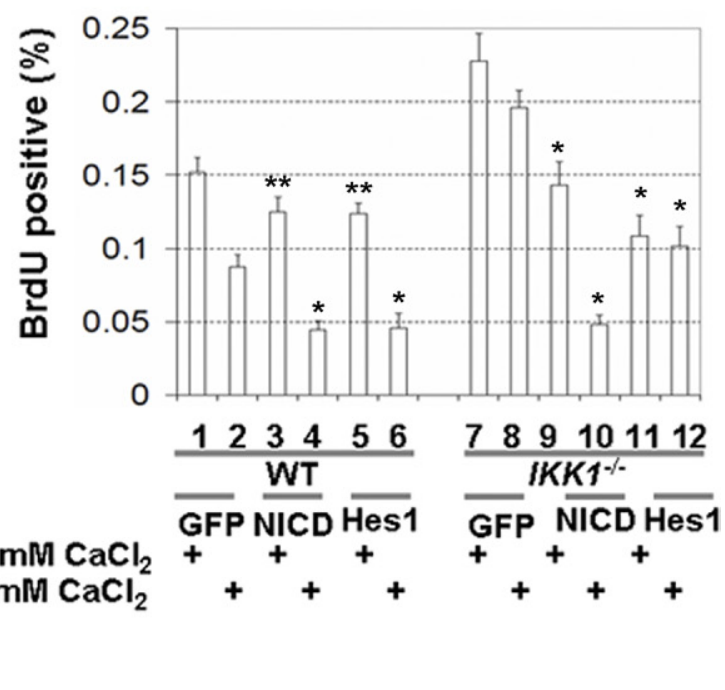

Figure 5. Overexpression of Hes1 inhibits $I k k 1^{-/-}$keratinocyte proliferation, but does not affect their differentiation. A-D: Tight junctions are formed in $I k k 1^{-1}$ keratinocytes expressing NICD, but not Hes1. Primary WT (A) and $I k k 1^{-/}$(B-D) keratinocytes were transduced with lentivirus-expressing NICD (C), Hes1 (D), or GFP as control (A and $\mathbf{B}$ ), and the tight junction formation was induced by $0.5 \mathrm{mmol} / \mathrm{L}$ calcium and visualized by $\mathrm{ZO}-1$ staining (red). WT and $I k k 1^{-/}$ keratinocytes transduced by lentiviruses expressing GFP, NICD/GFP, or Hes1/GFP were visualized by GFP fluorescence (green) and DAPI nuclear counterstaining (blue). Scale bar $=50 \mu \mathrm{m}$ (all images). E: Transcription of Hes1 and involucrin was measured by qPCR in primary WT or $I k k 1^{-/-}$keratinocytes transduced with lentivirus expressing NICD, or Hes1, or GFP as control, and treated with $0.5 \mathrm{mmol} / \mathrm{L}$ calcium for 48 hours. Data are reported as means \pm SD of three independent experiments. F: Quantitative results for the effects of NICD and Hes1 on cell proliferation shown by BrdU incorporation in WT and Ikk1 ${ }^{-/-}$keratinocytes. Analysis was performed on samples prepared from cells that had been treated as in $\mathbf{E}$, but incubated at 0.02 or 0.5 mmol/L calcium conditions. ${ }^{*} P<0.001$ and ${ }^{* * *} P<0.01$ compared with GFP transduced control samples; error bars indicate SD $(N=5)$. 
cells via Hes 1 remains controversial. ${ }^{22}$ To test this possibility, we transduced $l k k 1^{-1-}$ keratinocytes with lentiviruses expressing either Hes1 or NICD. The transduced cells could be visualized by GFP coexpressed by the same lentiviral vector. In contrast to NICD, Hes1 did not induce tight junction formation in $I k k 1^{-1-}$ keratinocytes, as demonstrated by ZO-1 staining (Figure 5, A-D). We showed that constitutive activation of Notch in WT and $1 \mathrm{kk} 1^{-1-}$ keratinocytes results in a dramatic induction of suprabasal markers, such as involucrin (Figure 4D). Although Hes 1 was ectopically expressed at the same level as NICD-induced Hes 1 expression (Figure 5E, columns 3 and 6 for involucrin versus columns 2 and 5 for Hes 1 ), involucrin expression was induced only by NICD (Figure $5 \mathrm{E}$, columns 2 and 5; compare with columns 3 and 6 for Hes1) in both WT and $I k k 1^{-1-}$ keratinocytes. This suggests that Notch promotes $\mid k k 1^{-1-}$ keratinocyte differentiation through a Hes1-independent mechanism. However, Hes1 had a similar inhibitory effect to NICD on the proliferation of $l \mathrm{kk} 1^{-1-}$ keratinocytes (Figure $5 \mathrm{~F}$ ).

\section{Discussion}

Epithelial development is a highly orchestrated differentiation process. After the differentiation program is launched, skin stem cells proliferate briefly for mitotic clonal expansion at the TA progenitor stage, then are committed to the next spinous cell type, from which the terminally differentiated granular and cornified cells are derived. Hyperplasia and differentiation blockage are associated with epidermal phenotypes in a variety of transgenic and knockout mouse models. ${ }^{35}$ Nonetheless, evidence suggests that proliferation and differentiation are connected but regulated differently. For example, transgenic expression of the cell cycle regulator Myc (also known as c-Myc), driven by the K14 promoter in stem cells and TA cells, leads to epidermal hyperproliferation but does not affect their differentiation. ${ }^{36}$ The Ikk1 lossof-function mutation affects both the proliferation and differentiation of keratinocytes, ${ }^{3-5}$ but the molecular mechanism by which IKK1 functions in regulating these processes is poorly understood.

As an epithelial stem cell marker, p63 plays a key role in skin morphogenesis by regulating epithelial development and differentiation. First, p63 is essential for commitment of the immature ectoderm to epidermal lineages through direct regulation of K14 gene expression. Second, it is important for the maintenance of the proliferative capacity of epidermal progenitor cells, performed mainly by $\Delta \mathrm{Np} 63$ isoforms. ${ }^{37}$ Consistent with both of these roles, p63 is strongly expressed in stem cells and TA cells located in the basal layer of normal epidermis. ${ }^{38}$ In our study, in contrast to WT skin, the $/ k k 1^{-1-}$ epidermis expressed p63 and K14 in both the basal and suprabasal layers. One possible reason for these suprabasal cells expressing basal cell-specific markers and proliferating is that the increased p63 expression in the suprabasal layer keeps the suprabasal cells in the progenitor stage. Consistent with this possibility, expression of $\Delta N p 63$ in transgenic lung epithelium induces squamous metaplasia and de novo $\mathrm{K} 5$ and $\mathrm{K} 14$ expression. ${ }^{14}$ In the present study, p63 knockdown in $/ k k 1^{-1-}$ keratinocytes not only inhibited cell proliferation, but also reduced $\mathrm{K} 14$ and $\mathrm{K} 5$ transcription in both WT and $l k \mathrm{k} 1^{-/-}$keratinocytes (data not shown). Our data suggest an intricate cross talk between p63 and IKK1, with p63 inducing expression of IKK1 during the early epidermal differentiation and IKK1 suppressing p63 expression to ensure further differentiation.

Notch signaling is another major pathway in the regulation of epidermal differentiation, and its activity is tightly controlled during epidermal differentiation and stratification. Notch signaling must be suppressed to maintain the proliferative capacity of stem cells and TA cells in the basal layer. When TA cells are ready to exit from the cell cycle to form spinous cells, Notch activity must be induced for proper differentiation of the spinous cells, and then later it must be down-regulated to allow spinous cells to differentiate into granular cells. ${ }^{25}$ Notch regulation of granular cell differentiation, however, is far more complicated. For example, transgenic mice expressing NICD driven by loricrin or K1 promoters show increased granular cell differentiation, ${ }^{23,24}$ but K14-driven NICD expression reduces granular cell differentiation. ${ }^{22}$ This discrepancy may due to timing of expression, or to the cell type controlled by the promoter, or to the level of the transgene expressed.

Our experiments showed that ectopic overexpression of NICD in Ikk1 mutant keratinocytes restored cell differentiation to form tight junctions upon high-calcium induction, suggesting that Notch signaling is a major downstream target of IKK1 in the promotion of epidermal differentiation. Furthermore, ectopic overexpression of Hes1, one of the major Notch targets, could not promote high calcium-induced tight junction formation, suggesting a Hes1-independent regulatory mechanism. Although in contrast to what was reported by Blanpain et $\mathrm{al},{ }^{22}$ our data are consistent with other reports. ${ }^{16,23,39}$ However, the overexpressed NICD was unable to support full terminal differentiation of $1 \mathrm{kk} 1^{-1-}$ keratinocytes into the granular cell types. There is a possibility that the constant overexpression of NICD inhibits the proper differentiation of granular cells. Alternatively, Notch may not be the only IKK1 target controlling epidermal differentiation, and other, as yet unidentified IKK1 targets may be required for full granular cell differentiation.

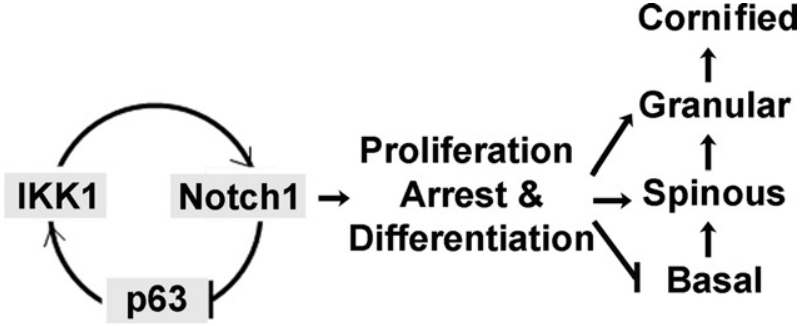

Figure 6. Schematic of molecular mechanisms in control of epidermal differentiation by IKK1. The major molecular signaling network (left) and the major epidermal epithelial cell types (right) are shown. The arrows in the diagram indicate activation or promotion and the bar-headed arrows indicate inhibition. Maintenance of the basal layer is controlled in part by p63, which maintains K14 expression and proliferation. In addition, p63 activates Ikk1 expression, which induces Notch1 expression in the spinous layer for further suprabasal epidermis differentiation. The induced Notch activity inhibits p63 expression by negative feedback. 
In summary, our data demonstrate a role for IKK1 in the developing epidermis and indicate that the underlying molecular mechanism is linked to the Notch1 signaling pathway. A working model for these molecular networks and their potential functional stages is presented in Figure 6. Briefly, the epidermal stem cells and TA cells in the basal layer express p63, which is required for the epidermal specification and proliferation of the basal cells; p63 also induces expression of $\mid k k 1$, which rapidly induces Notch1 expression. The enhanced Notch activity in the spinous cells inhibits p63 expression, thus switching off expression of $\mathrm{K} 5$ and $\mathrm{K} 14$ during the transition from the basal layer to the spinous layer. Moreover, the newly activated Notch inhibits proliferation and triggers suprabasal expression of $\mathrm{K} 10, \mathrm{~K} 1$, and involucrin for further spinous cell maturation and granular cell differentiation.

\section{Acknowledgment}

We thank Dr. Doug Dean for valuable discussion.

\section{References}

1. Fuchs E, Raghavan S: Getting under the skin of epidermal morphogenesis. Nat Rev Genet 2002, 3:199-209

2. Koster MI, Roop DR: Mechanisms regulating epithelial stratification. Annu Rev Cell Dev Biol 2007, 23:93-113

3. Hu Y, Baud V, Delhase M, Zhang P, Deerinck T, Ellisman M, Johnson $\mathrm{R}$, Karin M: Abnormal morphogenesis but intact IKK activation in mice lacking the IKKalpha subunit of IkappaB kinase. Science 1999, 284: $316-320$

4. Li Q, Lu Q, Hwang JY, Buscher D, Lee KF, Izpisua-Belmonte JC, Verma IM: IKK1-deficient mice exhibit abnormal development of skin and skeleton. Genes Dev 1999, 13:1322-1328

5. Takeda K, Takeuchi O, Tsujimura T, Itami S, Adachi O, Kawai T, Sanjo H, Yoshikawa K, Terada N, Akira S: Limb and skin abnormalities in mice lacking IKKalpha. Science 1999, 284:313-316

6. Hu Y, Baud V, Oga T, Kim KI, Yoshida K, Karin M: IKKalpha controls formation of the epidermis independently of NF-kappaB. Nature 2001, 410: 710-714

7. Sil AK, Maeda S, Sano Y, Roop DR, Karin M: IkappaB kinase-alpha acts in the epidermis to control skeletal and craniofacial morphogenesis. Nature 2004, 428:660-664

8. Descargues P, Sil AK, Sano Y, Korchynskyi O, Han G, Owens P, Wang XJ, Karin M: IKKalpha is a critical coregulator of a Smad4-independent TGFbeta-Smad2/3 signaling pathway that controls keratinocyte differentiation. Proc Natl Acad Sci USA 2008, 105:2487-2492

9. Liu B, Xia X, Zhu F, Park E, Carbajal S, Kiguchi K, DiGiovanni J, Fischer SM, Hu Y: IKKalpha is required to maintain skin homeostasis and prevent skin cancer. Cancer Cell 2008, 14:212-225

10. Candi E, Terrinoni A, Rufini A, Chikh A, Lena AM, Suzuki Y, Sayan BS, Knight RA, Melino G: p63 is upstream of IKK alpha in epidermal development. J Cell Sci 2006, 119:4617-4622

11. Mills AA, Zheng B, Wang XJ, Vogel H, Roop DR, Bradley A: p63 is a p53 homologue required for limb and epidermal morphogenesis. Nature 1999, 398:708-713

12. Yang A, Schweitzer R, Sun D, Kaghad M, Walker N, Bronson RT, Tabin C, Sharpe A, Caput D, Crum C, McKeon F: p63 is essential for regenerative proliferation in limb, craniofacial and epithelial development. Nature 1999, 398:714-718

13. Romano RA, Birkaya B, Sinha S: A functional enhancer of keratin14 is a direct transcriptional target of deltaNp63. J Invest Dermatol 2007, 127: 1175-1186

14. Romano RA, Ortt K, Birkaya B, Smalley K, Sinha S: An active role of the DeltaN isoform of p63 in regulating basal keratin genes $\mathrm{K} 5$ and K14 and directing epidermal cell fate. PLoS One 2009, 4:e5623

15. Dotto GP: Crosstalk of Notch with p53 and p63 in cancer growth control. Nat Rev Cancer 2009, 9:587-595
16. Nguyen BC, Lefort K, Mandinova A, Antonini D, Devgan V, Della Gatta G, Koster MI, Zhang Z, Wang J, Tommasi di Vignano A, Kitajewski J, Chiorino G, Roop DR, Missero C, Dotto GP: Cross-regulation between Notch and p63 in keratinocyte commitment to differentiation. Genes Dev 2006, 20:1028-1042

17. Bray SJ: Notch signalling: a simple pathway becomes complex. Nat Rev Mol Cell Biol 2006, 7:678-689

18. Okuyama R, Nguyen BC, Talora C, Ogawa E, Tommasi di Vignano A, Lioumi M, Chiorino G, Tagami H, Woo M, Dotto GP: High commitment of embryonic keratinocytes to terminal differentiation through a Notch1-caspase 3 regulatory mechanism. Dev Cell 2004, 6:551-562

19. Rangarajan A, Talora C, Okuyama R, Nicolas M, Mammucari C, Oh H, Aster JC, Krishna S, Metzger D, Chambon P, Miele L, Aguet M, Radtke F, Dotto GP: Notch signaling is a direct determinant of keratinocyte growth arrest and entry into differentiation. EMBO J 2001, 20:3427-3436

20. Lowell S, Jones P, Le Roux I, Dunne J, Watt FM: Stimulation of human epidermal differentiation by delta-notch signalling at the boundaries of stem-cell clusters. Curr Biol 2000, 10:491-500

21. Yamamoto N, Tanigaki K, Han H, Hiai H, Honjo T: Notch/RBP-J signaling regulates epidermis/hair fate determination of hair follicular stem cells. Curr Biol 2003, 13:333-338

22. Blanpain C, Lowry WE, Pasolli HA, Fuchs E: Canonical notch signaling functions as a commitment switch in the epidermal lineage. Genes Dev 2006, 20:3022-3035

23. Moriyama M, Durham AD, Moriyama $H$, Hasegawa $K$, Nishikawa $S$, Radtke F, Osawa M: Multiple roles of Notch signaling in the regulation of epidermal development. Dev Cell 2008, 14:594-604

24. Uyttendaele H, Panteleyev AA, de Berker D, Tobin DT, Christiano AM: Activation of Notch1 in the hair follicle leads to cell-fate switch and Mohawk alopecia. Differentiation 2004, 72:396-409

25. Nickoloff BJ, Qin JZ, Chaturvedi V, Denning MF, Bonish B, Miele L: Jagged-1 mediated activation of notch signaling induces complete maturation of human keratinocytes through NF-kappaB and PPARgamma. Cell Death Differ 2002, 9:842-855

26. Lefort K, Dotto GP: Notch signaling in the integrated control of keratinocyte growth/differentiation and tumor suppression. Semin Cancer Biol 2004, 14:374-386

27. Dotto GP: Notch tumor suppressor function. Oncogene 2008, 27:51155123

28. Demehri S, Turkoz A, Kopan R: Epidermal Notch1 loss promotes skin tumorigenesis by impacting the stromal microenvironment. Cancer Cell 2009, 16:55-66

29. Tiscornia G, Singer O, Verma IM: Design and cloning of lentiviral vectors expressing small interfering RNAs. Nat Protoc 2006, 1:234-240

30. Tiscornia G, Singer O, Verma IM: Production and purification of lentiviral vectors. Nat Protoc 2006, 1:241-245

31. Mammucari C, Tommasi di Vignano A, Sharov AA, Neilson J, Havrda MC, Roop DR, Botchkarev VA, Crabtree GR, Dotto GP: Integration of Notch 1 and calcineurin/NFAT signaling pathways in keratinocyte growth and differentiation control. Dev Cell 2005, 8:665-676

32. Koster MI, Roop DR: Transgenic mouse models provide new insights into the role of p63 in epidermal development. Cell Cycle 2004, 3:411-413

33. Pummi K, Malminen M, Aho H, Karvonen SL, Peltonen J, Peltonen S: Epidermal tight junctions: ZO-1 and occludin are expressed in mature, developing, and affected skin and in vitro differentiating keratinocytes. J Invest Dermatol 2001, 117:1050-1058

34. Yuki T, Haratake A, Koishikawa H, Morita K, Miyachi Y, Inoue S: Tight junction proteins in keratinocytes: localization and contribution to barrier function. Exp Dermatol 2007, 16:324-330

35. Mack JA, Anand S, Maytin EV: Proliferation and cornification during development of the mammalian epidermis. Birth Defects Res C Embryo Today 2005, 75:314-329

36. Arnold I, Watt FM: c-Myc activation in transgenic mouse epidermis results in mobilization of stem cells and differentiation of their progeny. Curr Biol 2001, 11:558-568

37. Senoo M, Pinto F, Crum CP, McKeon F: p63 Is essential for the proliferative potential of stem cells in stratified epithelia. Cell 2007, 129:523-536

38. Koster MI, Kim S, Mills AA, DeMayo FJ, Roop DR: p63 is the molecular switch for initiation of an epithelial stratification program. Genes Dev 2004, 18:126-131

39. Kolev V, Mandinova A, Guinea-Viniegra J, Hu B, Lefort K, Lambertini C, Neel V, Dummer R, Wagner EF, Dotto GP: EGFR signalling as a negative regulator of Notch1 gene transcription and function in proliferating keratinocytes and cancer. Nat Cell Biol 2008, 10:902-911 